\title{
Numerical investigations of vortex formation on a generic multiple-swept-wing configuration
}

\author{
D. Sedlacek $k^{1}$ - S. Biechele ${ }^{1} \cdot$ C. Breitsamter ${ }^{1}$
}

Received: 1 April 2021 / Revised: 4 October 2021 / Accepted: 10 November 2021 / Published online: 8 December 2021

(c) The Author(s) 2021

\begin{abstract}
For an improvement of the flight stability characteristics of high-agility aircraft, the comprehension of the vortex development, behavior and break down is important. Therefore, numerical investigations on low aspect ratio, multiple-swept-wing configurations are performed in this study to analyze the influence of the numerical method on the vortex formation. The discussed configurations are based on a triple- and double-delta wing planform. Unsteady Reynolds-averaged Navier-Stokes (URANS) simulations and delayed detached eddy simulations (DDES) are performed for both configurations. The simulations are executed at $\operatorname{Re}=3.0 \times 10^{6}$, symmetric freestream conditions, and an angle of attack of $\alpha=16^{\circ}$, for consistency with reference wind tunnel data. For the triple-delta-wing configuration, the results of the DDES show a satisfying accordance to the experiments compared to URANS, especially for the flow field and the pitching moment coefficient. For the doubledelta-wing configuration, the URANS simulation provides reliable results with low deviation of the aerodynamic coefficients and high precision for the flow field development with respect to the experimental data.
\end{abstract}

Keywords Hybrid-delta wing · Vortex interaction · Delayed detached eddy simulation

\section{List of symbols}

$\alpha \quad$ Angle of attack $\left({ }^{\circ}\right)$

$\beta \quad$ Angle of sideslip $\left({ }^{\circ}\right)$

$\gamma \quad$ Roll angle $\left({ }^{\circ}\right)$

$\Lambda \quad$ Aspect ratio (-)

$\varphi \quad$ Leading-edge sweep $\left({ }^{\circ}\right)$

$\omega \quad$ Axial vorticity (1/s)

$C_{\mathrm{D}} \quad$ Drag coefficient (-)

$C_{\mathrm{L}} \quad$ Lift coefficient (-)

$C_{Y} \quad$ Side force coefficient (-)

$C_{m x} \quad$ Rolling moment coefficient (-)

$C_{m y} \quad$ Pitching moment coefficient (-)

$C_{m z} \quad$ Yawing moment coefficient (-)

$C_{p} \quad$ Pressure coefficient (-)

$c_{\mathrm{r}} \quad$ Root cord (m)

$d \quad$ Seeding diameter (m)

$f_{\mathrm{m}} \quad$ Measuring frequency $(\mathrm{Hz})$

$l_{i} \quad$ Length of model section $i\left(\mathrm{~m}^{2}\right)$

$l_{\mu} \quad$ Mean aerodynamic chord (m)

$l_{\mathrm{Re}} \quad$ Reference length for Reynolds number (m)

D. Sedlacek

dominik.sedlacek@tum.de

1 Technical University Munich, Boltzmannstr. 15, 85748 Garching, Germany

$\begin{array}{ll}\text { Ma } & \text { Mach number }(-) \\ n_{\text {Prism }} & \text { Number of prismlayer }(-) \\ p_{t} & \text { Total pressure }(\mathrm{Pa}) \\ p_{t, \infty} & \text { Freestream total pressure }(\mathrm{Pa}) \\ Q & \text { Q-criterion }(-) \\ \operatorname{Re} & \text { Reynolds number }(-) \\ s & \text { Wing half span }(\mathrm{m}) \\ S_{\text {ref }} & \text { Wing reference area }\left(\mathrm{m}^{2}\right) \\ \Delta t & \text { Unsteady time step }(\mathrm{s}) \\ t_{\Delta m} & \text { Acquisition time }(\mathrm{s}) \\ U_{\infty} & \text { Freestream velocity }(\mathrm{m} / \mathrm{s}) \\ u, v, w & \text { Flow velocity in } x, y, \text { and } z \text { directions }(\mathrm{m} / \mathrm{s}) \\ x, y, z & \text { Body-fixed Cartesian coordinates }(\mathrm{m}) \\ y^{+} & \text {Non-dimensional wall distance }(\mathrm{m})\end{array}$

\section{Introduction}

High-agility aircraft often require an operation at extreme flight conditions, including the need for effective supersonic cruise and high maneuverability at subsonic conditions [11]. These configurations typically have low-aspectratio wings with medium to high sweep angles and small leading-edge radii. For these wing planforms, the flow tends to separate near the leading edge at low angles of attack 
and forms a leading-edge vortex (LEV). The LEV causes a suction peak on the wing surface and induces a nonlinear lift increase. At higher angles of attack, the fully developed LEV breaks down, the cross section of the vortex increases and a transient flow field develops downstream of the breakdown location. With further increase in angle of attack, the breakdown location moves upstream [12]. In the wake of the vortex breakdown, quasi-periodic velocity oscillations develop from helical mode instabilities. For slender wings, with leading-edge-sweep angles, $\varphi$, greater than $60^{\circ}$, the vortex core has a jet-type structure and the breakdown is abrupt due to a strong adverse pressure rise [2]. The vortex breakdown exhibits a reverse flow and has a major impact on flight stability characteristics. For non-slender and semislender wings, $50^{\circ}<\varphi<60^{\circ}$, the vortex core transitions to a wake-type structure at moderate angles of attack. The vortex breakdown, for this kind of wing planform, is a gradual transition with a less abrupt expansion of the vortex core and a moderate adverse pressure gradient [9]. Due to the less abrupt breakdown, the flight stability characteristics show a more stable behavior for non-slender-wing planforms.

Configurations with multiple-swept leading edges exhibit a considerably more complex flow field. With different swept leading-edge sections, multiple vortices can develop and form a vortex system. For double-delta-wing configurations, two vortices develop at small angles of attack and exist next to each other up to the trailing edge. For higher angles of attack, the vortices join and start rotating around each other. With further increase in angle of attack, the joined vortices burst. The vortex behavior is strongly dependent on the leading-edge sweeps and the position of the change between different swept leading-edge sections [3].

Pfnür and Breitsamter investigated the flow field and flight stability characteristics for double- and triple-deltawing configurations [17-19]. These configurations are similar to those investigated in the present study. The investigations show that the inboard vortex (IBV) is dominating the breakdown characteristics with different abruptness for non-slender and slender wing planforms. The second vortex developing in the midboard section, the so-called midboard vortex (MBV) is strongly influenced by the behavior of the IBV. The non-slender, triple-delta-wing configuration exhibits smoother instability onsets and smaller instability magnitudes, compared to the slender, double-delta-wing configuration [19].

Further comprehension of vortex formation, interaction and bursting is needed and computational fluid dynamics (CFD) represents an emerging solution toward establishing a better understanding of vortex flows and their effects. Since the beginning of numerical analysis for delta wings, the results have become increasingly precise. Morton [15] investigated the flow field of a delta wing with a sweep angle of $70^{\circ}$ using the detached eddy simulation (DES) and RANS methods. An unstructured mesh with $2.47 \times 10^{6}$ cells was used. The DES simulations achieved good agreement with the experimental data. For the RANS solution with the one equation model of Spalart-Allmaras (SA) [24], difficulties were observed in resolving the vortex bursting features. Adding a rotation correction (RC) [22] led to better agreement with the experimental data, especially in the vortex breakdown region [15].

Hitzel [10] performed simulations to analyze the flow field of the F-16XL aircraft that is comparable to the triple-delta-wing configuration in the present research. The turbulence models considered include the one-equation Spalart-Allmaras turbulence model with and without RC, as well as the $\mathrm{k}-\omega$ model. All models produced similar results for the pressure distribution and provided a good agreement with the flight test measurements. For non-slenderwing planforms, modeling issues arise in characterizing the separation and formation of the leading-edge vortex, as well as the breakdown behavior [14]. Therefore, future investigations are required to understand the influence of numerical methods, turbulence models, and grids.

In this study, data of CFD and wind tunnel simulations for force and moment measurements and particle image velocimetry (PIV) are compared for two wing configurations to demonstrate the efficacy of two turbulence modelling approaches. Unsteady Reynolds-averaged Navier-Stokes (URANS) simulations and delayed detached eddy simulations (DDES) are performed. Turbulence is modelled with the Spalart-Allmaras one-equation model in negative form with rotation correction (SARC-neg). The simulations were conducted at $\operatorname{Re}=3.0 \times 10^{6}$ and $\mathrm{Ma}=0.15$, symmetric freestream conditions and an angle of attack of $\alpha=16^{\circ}$. This angle of attack is chosen because previous studies [17, 19] have shown that the flow field at this angle of attack includes several vortex flow stages along the wing surface, namely LEV formation, dual LEV interference, interaction and merging as well as breakdown of merged leading-edge vortices.

\section{Configurations}

In this study, a generic wing-fuselage configuration with exchangeable wing planforms is considered. The configuration is the subject of a cooperation with Airbus Defence and Space (Airbus DS) and is embedded in the NATO STO AVT-316 task group. Attention is particularly paid to the following two configurations. The examined configurations, Fig. 1, are a triple-delta-wing configuration the so-called NA1 W1 displayed on the left hand side and the double-delta-wing configuration NA1 W2 on the right hand side. The NA1 W1 consists of three wing segments of different leading-edge sweep: The front part with a 

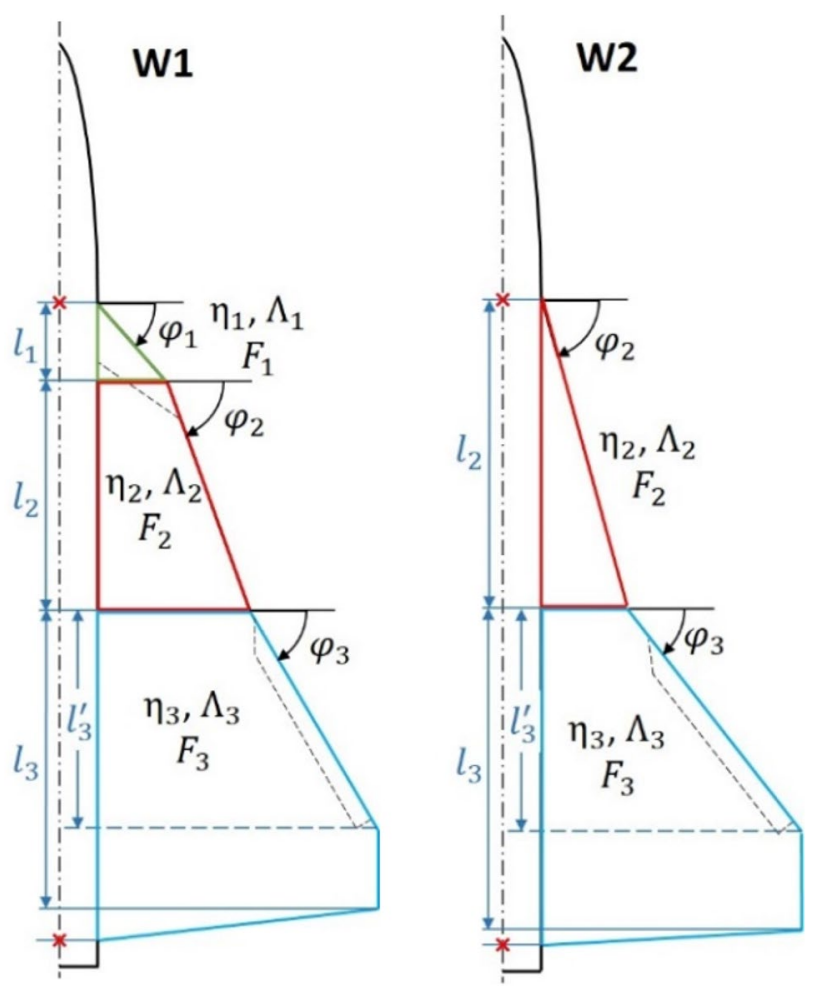

\section{Numerical methods and grid}

Main points on the set-up of the computational grid and numerical parameters are given in the following.

\subsection{Grid}

The grid, Fig. 2, was created with the grid generator Centaursoft and complied with the guideline of Cummings et al. [6]. Attention was paid to a similar grid structure for different configurations. Usually the model is created with a prism grid near the surface, so the surface grid consists of triangular elements. At critical points such as the leading and trailing edge, the surface grid consists of a structured grid. The farfield represents the outer frame of the grid and was chosen to be sufficiently large with a diameter of 50 times the model size so that the influence of the farfield boundary conditions on the model flow was minimized.

For optimal resolution of the wall boundary layer, the prism layer has an unstructured character on the surface and a structured character normal to the surface. The height of the prism layer was generally set by the number of layers, $n_{\text {Prism }}=33$, and a stretching ratio of 1.25 . These specifications were further refined by sources in important areas. An unstructured tetrahedron grid is used as the main cell shape, this connects directly to the prism layer and completely fills the space enclosed by the farfield plane of symmetry and model. For an improved resolution of the vortex system, a block of structured hexahedral elements is also inserted above the wing. For the connection between the hexahedron block and the tetrahedron grid, pyramids were used. The grid was additionally refined in defined areas, such as the leading edge and in the vortex field. The distance in wall units is $y^{+}<1.0$ from the wing's surface except in a small region near the wing root.

Table 1 Geometrical parameters of the NA1 W1 and NA1 W2 configurations

\begin{tabular}{llll}
\hline & & NA1 W1 & NA1 W2 \\
\hline$c_{\mathrm{r}}$ & $(\mathrm{m})$ & 0.802 & 0.802 \\
$s$ & $(\mathrm{~m})$ & 0.417 & 0.367 \\
$S_{\text {ref }}$ & $\left(\mathrm{m}^{2}\right)$ & 0.329 & 0.266 \\
$\Lambda$ & - & 0.15 & 0.16 \\
$l_{\mu}$ & $(\mathrm{m})$ & 0.468 & 0.426 \\
$l_{1} / c_{\mathrm{r}}$ & - & 0.125 & - \\
$l_{2} / c_{\mathrm{r}}$ & - & 0.350 & 0.475 \\
$l_{3} / c_{\mathrm{r}}$ & - & 0.475 & 0.475 \\
$\varphi_{1}$ & $\circ$ & 52 & - \\
$\varphi_{2}$ & $\circ$ & 75 & 75 \\
$\varphi_{3}$ & $\circ$ & 52 & 52 \\
\hline
\end{tabular}

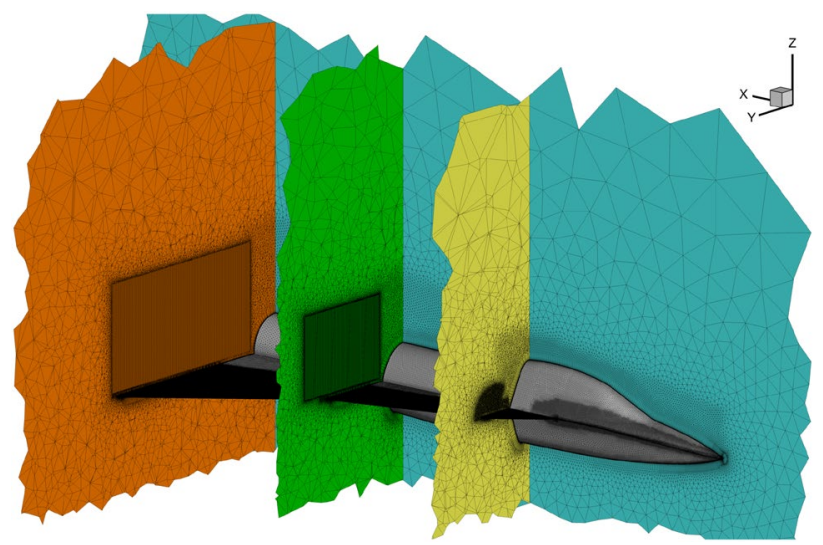

Fig. 2 Overview of the grid structure for NA1 W1 


\subsection{Numerical setup}

For the flow simulations, the incompressible Navier-Stokes equations with a finite volume method were solved using the DLR-Tau-Code. Two different numerical methods are used. TAU uses a dual time step method for unsteady simulations. The physical time step is implicitly discretized. The next physical time step is determined iteratively using explicit pseudo time steps. For the URANS solution, the turbulence was modelled by the one equation model of Spalart-Allmaras with rotation correction $[1,23,24]$. The unsteady time step $\delta_{t}=2 \times 10^{-4} \mathrm{~s}$ for both configurations was calculated based on [7]:

$\delta_{t}=\frac{\delta_{t}^{*} \times l_{\mu}}{U_{\infty}}$

For this purpose, a characteristic time step of $\delta_{t}^{*}=0.002 \mathrm{~s}$ is recommended. Unsteady inner iterations per time step were limited to 50-300.

As second method, a DDES was conducted. The idea of the DES, combining URANS and large eddy simulations (LES), is to model the areas close to the wall and areas with smaller element length than the turbulent length scales with a RANS model. The LES model is only used in regions fine enough for LES calculations. This significantly reduces the computing effort and increases the accuracy of the RANS model. The DDES is a modification introduced by Spalart $[26,27]$. It maintains the usage of RANS in the boundary layer even if the grid spacing parallel to the wall is smaller than the boundary layer, so the switching is delayed in boundary layers. Spalart [25] gives a guideline for the unsteady DDES time step:

$\delta_{t}=\frac{\Delta}{u_{\max }}$

For a minimum element size $\Delta=9 \times 10^{-4} \mathrm{~m}$ and a maximum velocity of $u_{\max }=130 \mathrm{~m} / \mathrm{s}$, the unsteady time step for DDES can be defined as $\delta_{t}=7 \times 10^{-6} \mathrm{~s}$. and the computations converged well within 200 inner iterations per physical time step.

To confirm a sufficient discretization of the time step, in Table 2, the non-dimensional time step is compared

Table 2 Comparison of the physical time step for DES with several studies [4, 6, 8, 15] concerning similar configurations. For Cummings et al., the smallest time step of the time step study is presented. The time step used was chosen significantly lower than in comparable studies. Therefore, the selected time step achieves a high level of time discretization and a solution variability due to the time step can be excluded.

A second-order central scheme as discretization type was employed. The results of the converged DDES solutions are presented in this study. The shown results represent the averaged values of the flow field. The graphs and figures presented in the following study refer to the mean values of the time-accurate CFD computations.

\subsection{Grid resolution}

A grid resolution study is performed with six different grids for both configurations at an angle of attack of $\alpha=16^{\circ}$. The grid resolution for the NA1 W2 is shown in Fig. 3. The number of grid elements range between $12 \times 10^{6}-110 \times 10^{6}$ elements. The lift coefficient $C_{\mathrm{L}}$, in black, the drag coefficient $C_{\mathrm{D}}$, in green, as well as the pitching moment coefficient $C_{m y}$, in blue, are depicted. The solid lines represent the experimental data and the dash-dot line the CFD data. For the meshes with a higher number of elements than $52 \times 10^{6}$, the coefficients show no major changes. Especially, the pitching moment coefficient is stable. The mesh for the NA1 W1 configuration has the same structure and shows the same grid resolution behavior. Therefore, the meshes with a number of elements of $52 \times 10^{6}$ are chosen for further simulations.

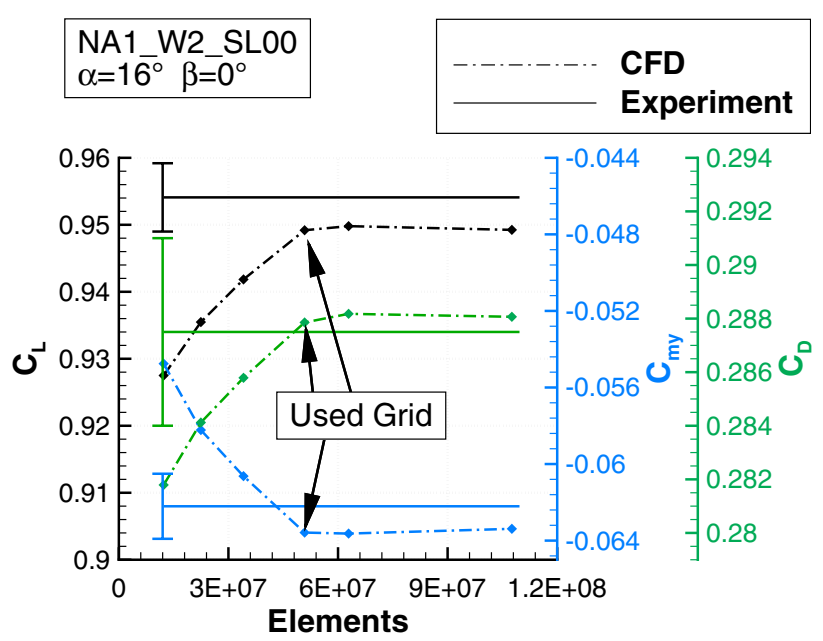

Fig. 3 Lift coefficient $C_{\mathrm{L}}$, black, drag coefficient $C_{\mathrm{D}}$, green, and the pitching moment coefficient $C_{m y}$, blue, for grids with various numbers of elements at $\operatorname{Re}=3.0 \times 10^{6}$ and $\alpha=16^{\circ}$ for NA1 W2 configuration 


\section{Experimental methods}

The experiments were conducted in the wind tunnel A of the Technical University of Munich. It features an open test section with a cross section of $1.80 \mathrm{~m} \times 2.40 \mathrm{~m}$ and a length of $4.80 \mathrm{~m}$. The wind tunnel reaches a maximum velocity of $U_{\mathrm{WT}}=65 \mathrm{~m} / \mathrm{s}$ and provides a turbulence level below $0.4 \%$. A three component model support is used to set the angle of attack $\alpha$, the angle of sideslip $\beta$ and the roll angle $\gamma$. It can be adjusted for sideslip and roll angle within $\pm 90^{\circ}$. The possible range for the angle of attack is limited to $\alpha=0-40^{\circ}$.

The experimental methods and results of these measurements are also published in [17-19]. For the measurements, a freestream velocity of $U_{\infty}=48 \mathrm{~m} / \mathrm{s}$, a Reyolds number of $\operatorname{Re}=3.0 \times 10^{6}$ based on the reference length $l_{\mathrm{Re}}=1 \mathrm{~m}$ was used. The following will be a short overview for most important parameters.

\subsection{Forces and moments}

An internal six-component strain-gauge balance was used to acquire the aerodynamic forces and moments. The maximum sustainable loads read $900 \mathrm{~N}, 450 \mathrm{~N}, 2500 \mathrm{~N}$ for axial, lateral, and normal forces, respectively. The maximum sustainable moments are $120 \mathrm{Nm}, 160 \mathrm{Nm}, 120 \mathrm{Nm}$ for rolling, pitching, and yawing moments, respectively. The forces and moments were measured with a sampling frequency $f_{\mathrm{m}}=800 \mathrm{~Hz}$ for a total acquisition time of $t_{\mathrm{m}}=10 \mathrm{~s}$. The accuracy of the aerodynamic coefficients for the applied test setup is presented in Table 3 with respect to repeatability.

The repeatability is defined as the standard deviation of the coefficients determined from several angle of attack polar measurements. The standard deviation was determined for every angle of attack and coefficient. The force and moment measurements presented in the following refer to the mean values of the acquired series of measurments per angle of attack.

\subsection{Particle image velocimetry}

A Stereo-PIV measurement system was used to measure the flow field above the wing in several crossflow sections. Thus, all three velocity components are obtained. For the

Table 3 Repeatability of measured force and moment coefficients

\begin{tabular}{ll}
\hline$\Delta C_{\mathrm{D}}$ & \pm 0.0035 \\
$\Delta C_{Y}$ & \pm 0.0012 \\
$\Delta C_{\mathrm{L}}$ & \pm 0.0051 \\
$\Delta C_{m x}$ & \pm 0.0005 \\
$\Delta C_{m y}$ & \pm 0.0017 \\
$\Delta C_{m z}$ & \pm 0.00042 \\
\hline
\end{tabular}

adjustment of the measured plane, the Stereo-PIV system was mounted on a three axis traversing system next to the wind tunnel test section, see Fig. 4a. For an alignment of the cameras and the laser sheet with the angle of attack of the wind tunnel model, the cameras and laser sheet can be rotated around the $Y$-axis of the traversing system. The two sCMOS cameras have a resolution of $2560 \times 2160$ pixel and were placed up- and downstream of the measurement plane with an angle of $60^{\circ}$. The sCMOS sensor planes were tilted by Scheimpflug adapters to meet the Scheimpflug criterion, cf. [20]. Seeding particles with a diameter of $\approx 1 \mu \mathrm{m}$ were infused into the flow. The cameras recorded 400 image pairs per cross section with a sampling frequency of $f_{\mathrm{m}}=15 \mathrm{~Hz}$. The presented results are the mean values determined by the 400 samples. The uncertainties of the mean velocity components were quantified to $\left|u_{\text {err }} / U_{\infty}\right|<0.06$ and $\left|v_{\text {err }} / U_{\infty}\right|=\left|w_{\text {err }} / U_{\infty}\right|<0.035$ [21]. Figure $4 \mathrm{~b}$ shows

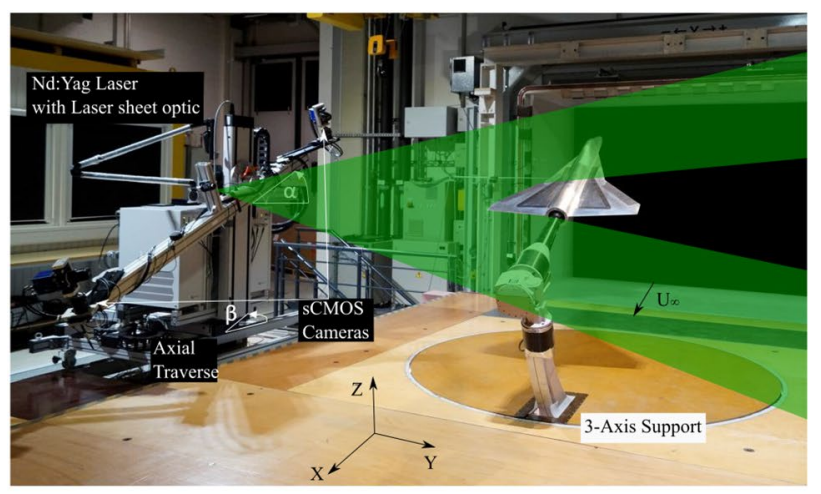

(a)

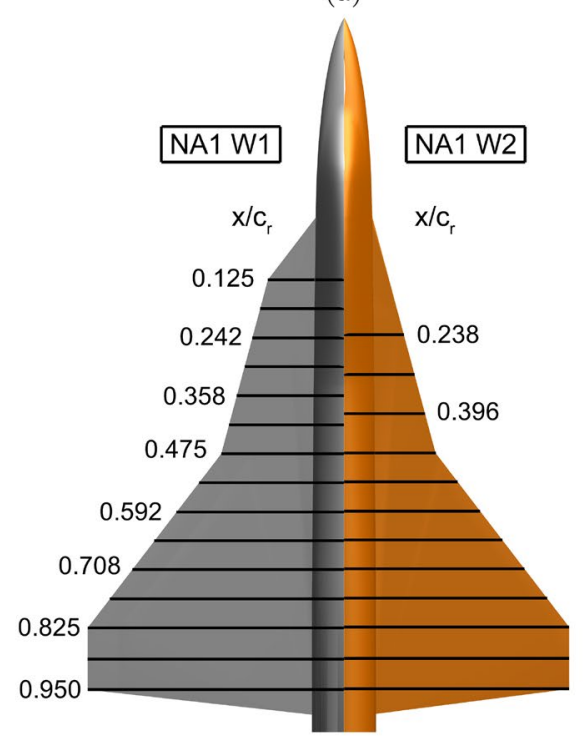

(b)

Fig. 4 a Stereo-PIV measurement setup and b measured crossflow sections [19] 
the position of the measured crossflow sections for both configurations.

\subsection{Oil flow pictures}

Surface streamline visualization is obtained applying oil flow tests. The model is painted or sprayed with a mixture of oil and color particles. If the model is exposed to the flow, the mixture is distributed by the flow and streamlines become visible on the model [16].

A mixture of cosmetic oil and fluorescent paint particles was used in these studies. The oil/pigment mass ratio defines the viscosity of the mixture. Investigations by Buzica and Breitsamter [5] on a low aspect ratio configuration at comparable freestream velocities indicate an oil/pigment mass ratio of about 2:1 for this type of flows. Intense skin friction gradients develop in a flow field with strong vortices. The heterogeneous flow field exhibits locally very high skin friction values below the vortex. In the inboard wing area and at the leading edge, the skin friction values are significantly lower. Therefore, different oil/pigment mixing ratios between 2:1 to 3:1 must be used for these wing areas, whereas a low viscosity mixture was applied near the leading edge and in some local areas at the rear wing section. The arising flow skin friction patterns were captured with a digital camera under black light conditions. The wing was covered by a glossy black foil with a thickness of about $100 \mu \mathrm{m}$ to obtain a better contrast between the mixture and the metallic wing surface. [19]

\section{Results}

Various experimental and numerical investigations were carried out for this study to be able to assess the accuracy of the numerical simulations depending on the flow configuration. It is particularly important to what extent cost efficient numerical methods resolve the flow and the aerodynamic coefficients with sufficient accuracy and for which cases more complex and costly methods are required.

In the following, the aerodynamic coefficients, the crossflow velocity fields and the flow close to the wall are compared for the respective configurations, and the differences and accuracy of the numerical methods are shown on the basis of the experimental results.

\subsection{Triple-delta wing-NA1 W1}

\subsubsection{Forces and moments}

The results of the force and moment measurements as well as the simulations are shown in Fig. 5. The lift coefficient is marked as square symbols and the pitching moment

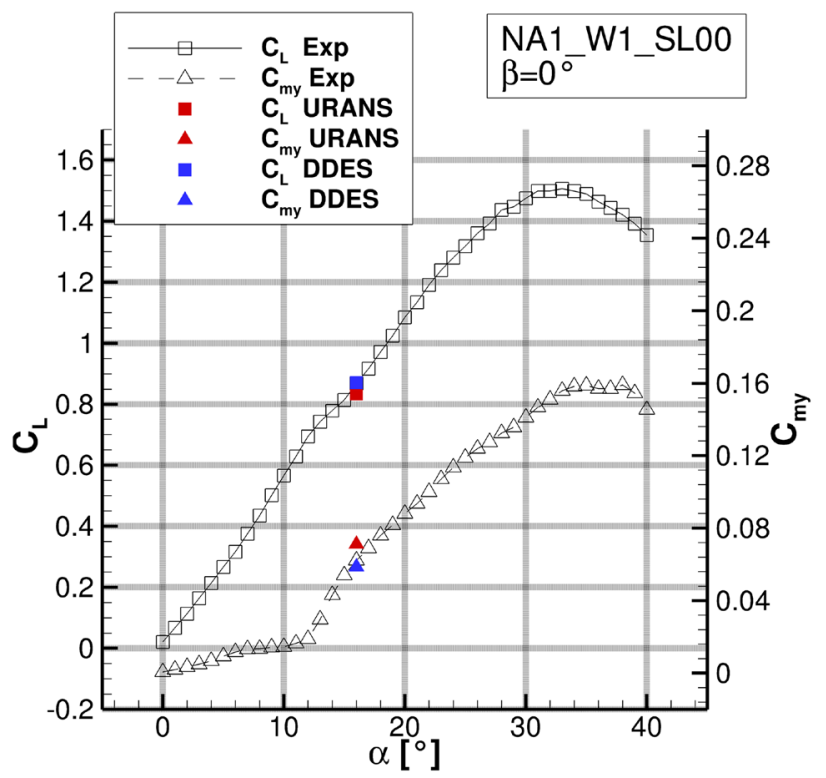

Fig. 5 Comparison of lift coefficient $C_{\mathrm{L}}$ and pitching moment coefficient $C_{m y}$ for NA1 W1 between experiments, URANS and DDES results at $\operatorname{Re}=3.0 \times 10^{6}$

Table 4 Absolute value and deviation of lift and pitching moment coefficient for $\alpha=16^{\circ}, \mathrm{Re}=3.0 \times 10^{6}$ for NA1 W1 configuration

\begin{tabular}{llllll}
\hline & \multicolumn{1}{l}{$C_{\mathrm{L}}$} & & \multicolumn{2}{l}{$C_{m y}$} \\
\cline { 2 - 3 } \cline { 5 - 6 } & - & $\%$ & & - & $\%$ \\
\hline EXP & 0.868 & - & 0.0621 & - \\
URANS & 0.834 & $-3.4 \%$ & & 0.0687 & $+10.63 \%$ \\
DDES & 0.867 & $+0.115 \%$ & & 0.0594 & $-4.34 \%$ \\
\hline
\end{tabular}

coefficient as triangular symbols. In addition, the experimental results are shown by black non-filled squares and triangles and the numerical results as symbols colored in red for URANS, and symbols colored in blue for DDES. A positive pitching moment coefficient causes a nose-up behavior of the configuration.

For the lift coefficient, the non-linear increase in lift typical for delta wings can already be seen beyond $\alpha \approx 8^{\circ}$. The maximum angle of attack is reached at $\alpha_{\max } \approx 32^{\circ}$ and $C_{\mathrm{L}, \max } \approx 1.55$. The course of the pitching moment coefficient increases significantly from an angle of attack larger than $\alpha \approx 12$. As the angle of attack increases, this leads to an increasing, unstable pitch up behavior.

A precise comparison of the coefficients and the percentage deviation from the experimental results for $\alpha=16^{\circ}$ is given in Table 4. As expected, the results of the DDES are, compared to the deviation of the URANS results, much closer to the experimental results. Nevertheless, the results of the URANS simulations show a sufficiently good 
agreement with the experimental data, especially regarding the lift coefficient.

\subsubsection{Flow field}

For the analysis of the flow field, the non-dimensional axial velocity $u / U_{\infty}$ in different cross sections at $\alpha=16^{\circ}$ is shown in Fig. 6 for the results of PIV experiments (left), the URANS simulations (center) and the DDES (right). The positions of the crossflow sections are shown in Fig. $4 \mathrm{~b}$. In the first section at $x / c_{\mathrm{r}}=0.125$, the inboard vortex, IBV, is represented by high velocities in the vortex core, $u / U_{\infty}=1.96$, for the PIV results, indicating a jettype vortex. The results of both numerical methods show a reduced vortex core velocity. In the URANS results, the core flow is already of reverse type, $u / U_{\infty}=-0.07$, and in the DDES solution the velocities are within the range of the freestream velocity, $u / U_{\infty}=0.98$. The flow field of both simulations indicate a wake-type vortex structure. For wake-type vortices, the axial velocities are stagnating or reversed and the flow around the core exhibits higher axial velocities of $u / U_{\infty}>1.5$ as well as rotational velocities of $v / U_{\infty}, w / U_{\infty}>1.5$.

At $x / c_{\mathrm{r}}=0.475$, all results show a wake-type IBV. The vortex core velocities of the URANS solution, $u / U_{\infty}=-0.18$, differs strongly from the DDES result, $u / U_{\infty}=0.91$, and PIV, $u / U_{\infty}=0.73$. In the surrounding area the URANS simulation shows a better agreement with the PIV results. The DDES solution shows slightly lower velocities and a smaller region of accelerated flow. The bursting onset is at $x / c_{\mathrm{r}} \approx 0.475$, cf. [19].
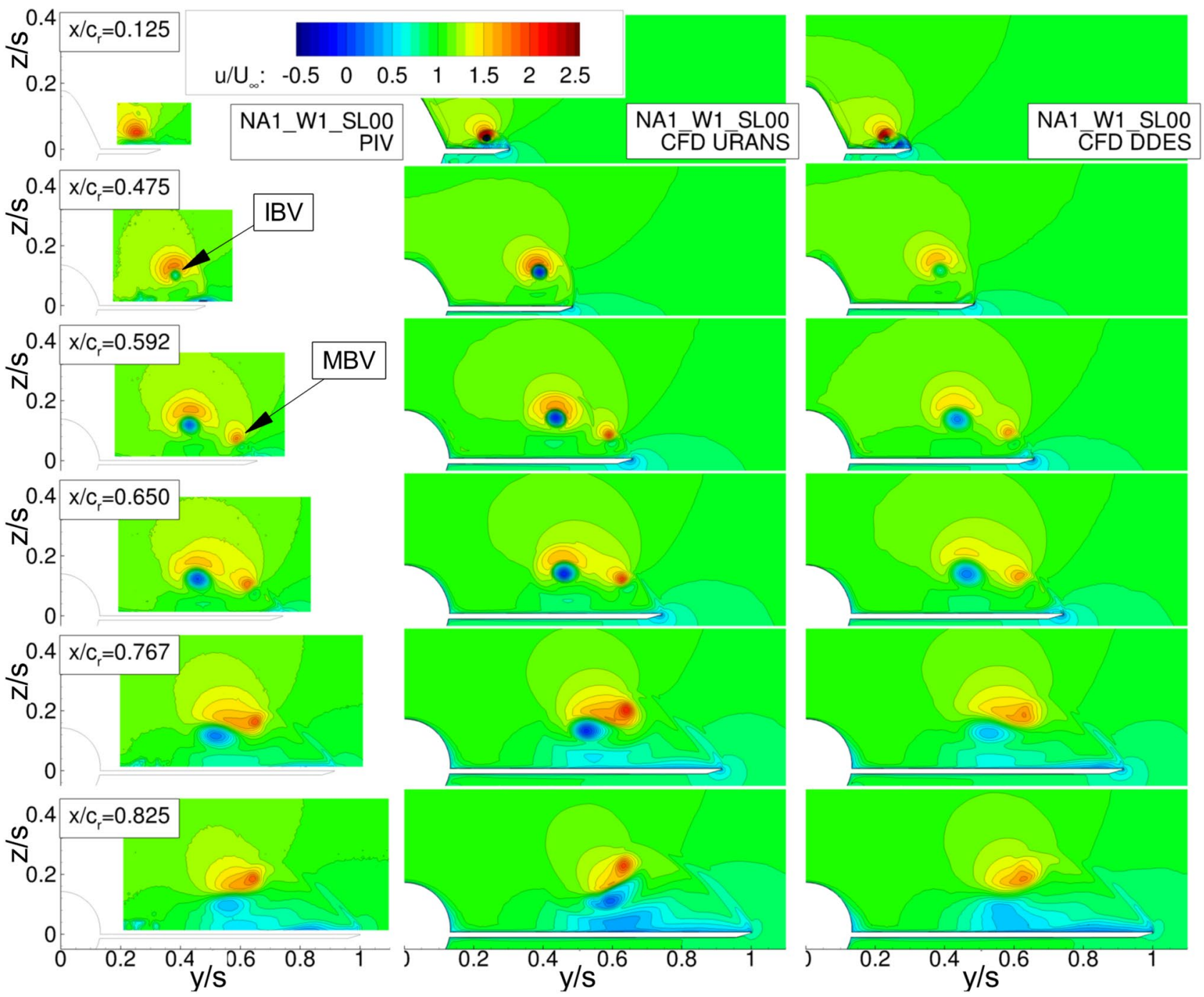

Fig. 6 PIV, URANS and DDES results of non-dimensional axial velocities $u / U_{\infty}$ at $\alpha=16^{\circ}$ and $\operatorname{Re}=3.0 \times 10^{6}$ for cross section planes ranging from $x / c_{\mathrm{r}}=0.125$ to $x / c_{\mathrm{r}}=0.825$ for NA1 W1 configuration 
The crossflow sections at $x / c_{\mathrm{r}}=0.592$ and $x / c_{\mathrm{r}}=0.650$ show the flow field downstream of the second change in wing sweep. At the non-slender leading edge, a second vortex develops, the so-called midboard vortex (MBV). The MBV represents a jet-type core flow and is shown to the right of the waketype IBV. At $x / c_{\mathrm{r}}=0.650$, the discrepancy between vortex core velocities of the URANS solution, $u / U_{\infty}=-0.04$, and the PIV results, $u / U_{\infty}=0.09$, in the vortex core is decreasing. The DDES indicates a too dissipative behavior regarding the surrounding flow field and the MBV core velocities, $u / U_{\infty}=1.48$. The MBV core velocities received with PIV is $u / U_{\infty}=1.78$ and with URANS, $u / U_{\infty}=1.88$.

At $x / c_{\mathrm{r}}=0.767$, the IBV is completely burst and no vortical structure is recognizable. The MBV has moved inboard und further away from the wing surface. The movement of the MBV differs slightly between the results. This is further intensified at $x / c_{\mathrm{r}}=0.825$. The position of the MBV in the URANS solution is the most inboard. DDES and PIV show a similar position of the velocity peak of the MBV.

In summary, compared to the PIV results, the MBV of the URANS data exhibits a slightly higher axial velocity, but is otherwise similar in shape and position. The DDES solution provides the flow quantities in the core of the IBV closer to the experimental data, but velocities of the vortex environment are shown slightly too small. Consequently, the vortex field is not optimally represented by both simulations, whereby the DDES solution is much closer to the PIV data than the URANS solution.

The representation of isosurfaces of the Q-criterion serves as an additional overview of the flow field. The Q-criterion defines a vortex as a connected fluid region with a positive second invariant of the velocity gradient for the identification of vortex structures. These are areas in which the amount of vortex strength is greater than the amount of shear rate. The Q-criterion is calculated by [13]:

$Q=\frac{1}{2}\left(u_{i, i}^{2}-u_{i, j} u_{j, i}\right)=-\frac{1}{2} u_{i, j} u_{j, i}=$

$\frac{1}{2}\left(\|\Omega\|^{2}-\|\mathbf{S}\|^{2}\right)>0$

$\Omega$ is the vorticity tensor and $\mathbf{S}$ represents the strain rate tensor. In Fig. 7, the isosurfaces of the non-dimensional Q criterion $Q^{*}=\frac{Q \times l_{\mu}^{2}}{U_{\infty}}=50$ colored by the total pressure ratio $p_{t} / p_{t, \infty}$ and the non-dimensional axial velocity $u / U_{\infty}=0$, in black, are illustrated. The positions of the flow field cross sections shown above, c.f. Fig. 6, are indicated by black lines on the model. The left side displays the DDES results and the right the isosurfaces of the URANS simulations. The IBV in the DDES simulation shows a slightly increasing diameter of the vortex core up to $x / c_{\mathrm{r}}=0.475$. Downstream, the expansion of the vortex core increases but shows still a

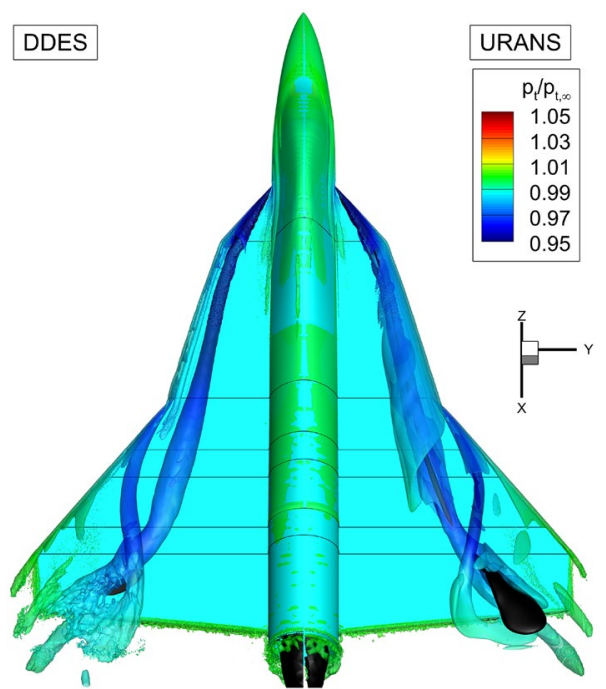

Fig. 7 Isosurfaces of the $Q^{*}$-criterion, $Q \times l_{\mu}^{2}=50$, colored with the total pressure ratio $p_{t} / p_{t, \infty}$, and $U / U_{\infty}=0^{\mu}$ (black) for DDES (left) and URANS (right) at $\alpha=16^{\circ}$ and $\operatorname{Re}=3.0 \times 10^{6}$ for NA1 W1 configuration

stable behavior. At $x / c_{\mathrm{r}}=0.825$, the IBV loses its structure and the breakdown process is completed.

The MBV separates from the leading edge and interacts with the IBV by rotating around the IBV. Due to the interaction the IBV is moved in outboard direction. In the front region the secondary vortex is visible close to the leading edge. In the DDES solution no regions with reverse flow occur. The isosurfaces display a slowly increasing pressure in the vortex core with a rising gradient downstream of $x / c_{\mathrm{r}}=0.45$.

The URANS simulations show similar results. However, the IBV core indicates a more unstable behavior and the shear layer is visible in the $Q^{*}$-isosurface. As previously discussed, the reverse flow in the IBV core is also visible, starting shortly downstream of the apex. The MBV isosurface, compared to the DDES result, indicates a more stable and stronger vortex due to a smaller core cross section and lower pressure. Furthermore, the turbulent DDES flow field, on the left side, downstream of $x / c_{\mathrm{r}}=0.825$ is significantly better resolved than in the URANS simulation.

In Fig. 8, the trajectory of the IBV vortex core is shown for URANS and DDES simulations. The position of the vortex core is determined by the highest level of the Q-criterion. The DDES result is displayed in black, the URANS one in blue. Additionally, the MBV trajectory is illustrated by a dashed line. The breakdown onset is marked at $x / c_{\mathrm{r}} \approx 0.475$. Upstream, the IBV is shown with a solid line, downstream with a dotted line. The IBV core positions differ only slightly in the front region. The IBV core position of the URANS simulation is marginally closer to the leading edge than the DDES core position. In summary, the numerical methods 


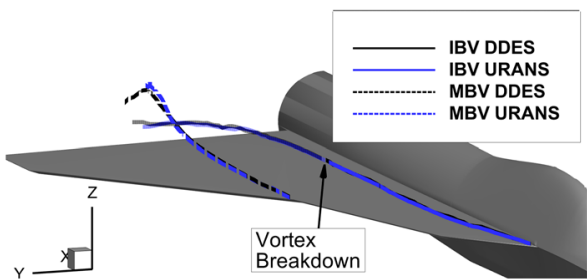

Fig. 8 Vortex core position for URANS (blue) and DDES (black) simulation determined by the $Q^{*}$-criterion at $\alpha=16^{\circ}$ and $\operatorname{Re}$ $=3.0 \times 10^{6}$ for NA1 $\mathrm{W} 1$ configuration

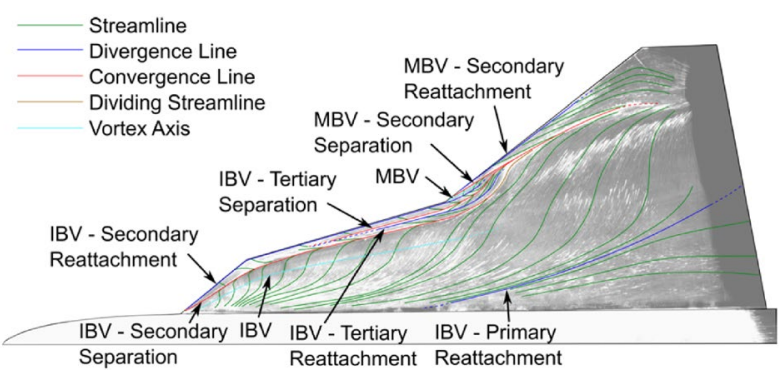

(a) Oil flow picture and inferred wall stream lines [19]
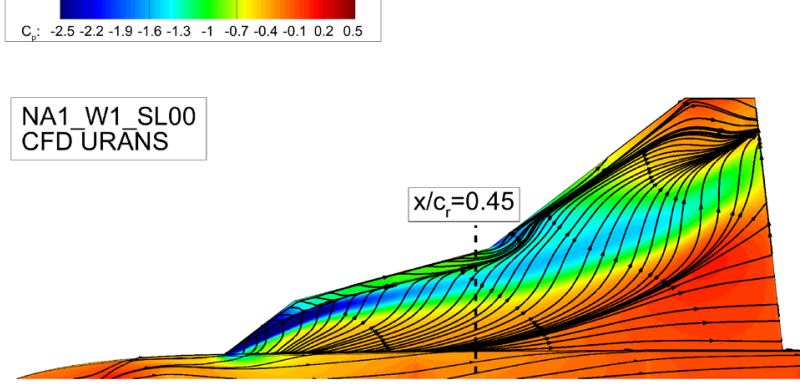

(b) Surface pressure distribution $C_{p}$ and skin friction lines of URANS solution

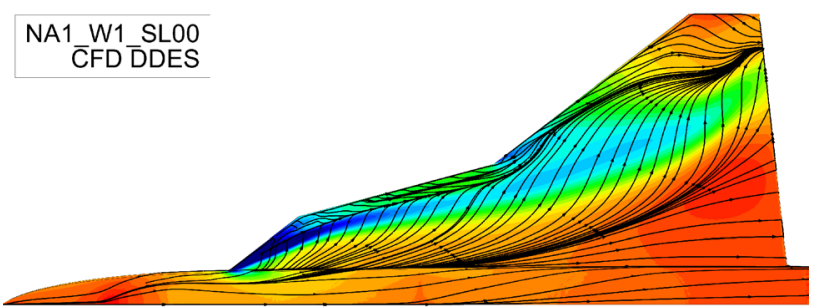

(c) Surface pressure distribution $C_{p}$ and skin friction lines of DDES solution

Fig. 9 Experimental and numerical results of the near-wall flow tests at $\alpha=16^{\circ}$ and $\mathrm{Re}=3.0 \times 10^{6}$ for NA1 W1 configuration

provide quite similar results regarding the vortex core positions in this case.

\subsubsection{Near-wall flow}

The near-wall flow of the NA1 W1 configuration is discussed by means of oil flow visualization and pressure distributions obtained by URANS and DDES, see Fig. 9. Figure 9a presents the skin friction patterns and streamline sketches at $\alpha=16^{\circ}$ and $\beta=0^{\circ}[19]$ for comparison with the numerical results. The most important sketches shown are streamlines in green, converging streamlines in red and diverging ones in blue. While converging streamlines indicate separation, diverging streamlines indicate reattachment. The position of the vortex axis is marked with light blue lines.

The trace of the IBV is characterized by strongly outboard deflected streamlines. Due to the sharp leading edge the IBV separation is fixed to the leading edge. The attachment line is located either too close to the wing root or on the fuselage to be observed in the front wing area. Close to the leading edge in the highly swept midboard section two separation lines are shown indicating a secondary and tertiary separation. The flow reattaches outboard of the secondary separation line near the leading edge.

Downstream of $x / c_{\mathrm{r}}=0.475$, the MBV and another secondary structure develop. The IBV secondary and tertiary separation lines merge with the MBV secondary separation line. Due to the detachment of the MBV from the wing surface the secondary separation line does not follow the leading edge. The vortex breakdown of the IBV is characterized by less deflected streamlines in the midboard wing area. The reattachment line is detectable starting at $x / c_{\mathrm{r}} \approx 0.475$ and further downstream it moves outboard [19].

The numerical results show approximately the same structure. But for the URANS simulation no tertiary structures can be observed and the secondary separation line of the MBV stays longer close to the leading edge.

In Fig. 9b, c, the distribution of the surface pressure coefficient $C_{p}$ is presented. The results for both numerical methods are comparable, which is expected due to the DDES switching to RANS in areas close to the wall. In the medium swept front section, the IBV suction peak is observable. A strong suction area forms between the leading edge and the trace of the vortex axis. At $x / c_{\mathrm{r}}=0.125$, the IBV is deflected downstream and the trace of the vortex axis runs parallel to the highly swept leading edge. In the DDES solution, a tertiary vortex structure is also observable in the pressure distribution. The IBV suction peak decreases in downstream direction. After the midboard change in sweep angle at $x / c_{\mathrm{r}}=0.475$, the IBV peak increases slightly and a second suction peak appears due to the MBV. The suction peaks merge and due to the MBV rotation around the IBV the overall suction area decreases.

In Fig. 10, the surface pressure distribution $C_{p}$ is shown for $x / c_{\mathrm{r}}=0.125,0.475,0.592,0.825$. The DDES results are indicated with a solid line and the URANS distributions are illustrated by a dashed line. In the first slice, at $x / c_{\mathrm{r}}=0.125$ in black, the vortex suction peak of the URANS simulation is significantly higher compared to the DDES result. This is a cause of the previously discussed higher pitch up moment 


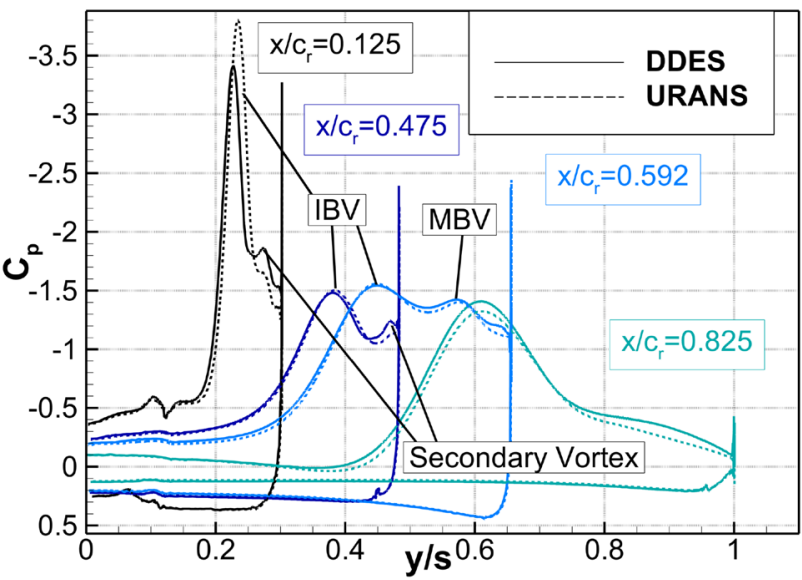

Fig. 10 Surface pressure distribution $C_{p}$ for selected sections at $\alpha=16^{\circ}$ and $\operatorname{Re}=3.0 \times 10^{6}$ for the NA1 W1 configuration

for the URANS results, Fig. 5. The slightly lower surface pressure in the rear area, $x / c_{\mathrm{r}}=0.825$, contributes to this effect. The secondary vortex can be recognized by a small suction tip near the leading edge. The peak is particularly pronounced in the high sweep area, $x / c_{\mathrm{r}}=0.125,0.475$, in the DDES results. In the URANS pressure distribution, this is hardly developed. At $x / c_{\mathrm{r}}=0.475$, the suction peak of the IBV has decreased by more than $60 \%$. Both methods are in good agreement disregarding the secondary vortex. At $x / c_{\mathrm{r}}=0.592$, the suction peak of the IBV has slightly increased due to the development of the MBV. Because of the rotation of the MBV, the vortex moves above the burst IBV at $x / c_{\mathrm{r}}=0.825$ and, therefore, only one suction peak is visible.

For a better understanding of the secondary vortex structures and the resolution in the DDES and URANS simulations, Fig. 11 shows the non-dimensional axial vorticity $\omega l_{\mu} / U_{\infty}$ at $x / c_{\mathrm{r}}=0.45$, including the flow streamlines (solid). Furthermore, the surface pressure distribution as well as the skin friction lines (dash-dotted) are illustrated. In Fig. 11a, the negative non-dimensional axial vorticity close to the surface indicates a clockwise rotating flow. For the URANS skin friction lines, a secondary separation of the IBV is visible. The streamlines show a strong curvature of the flow but no actual secondary vortex is visible. However, for the DDES results, Fig. 11b, higher negative vorticity levels are present and the streamlines form a distinct secondary vortex. The skinfriction lines indicate a similar secondary separation but due to the development of the secondary vortex a further separation and reattachment line is visible. The separation line also belongs to a tertiary separation. The tertiary reattachment is still observable. The development of the secondary vortex causes the reattachment line to move closer to the leading edge and causes a significant increase in the secondary suction peak, Fig. 10.

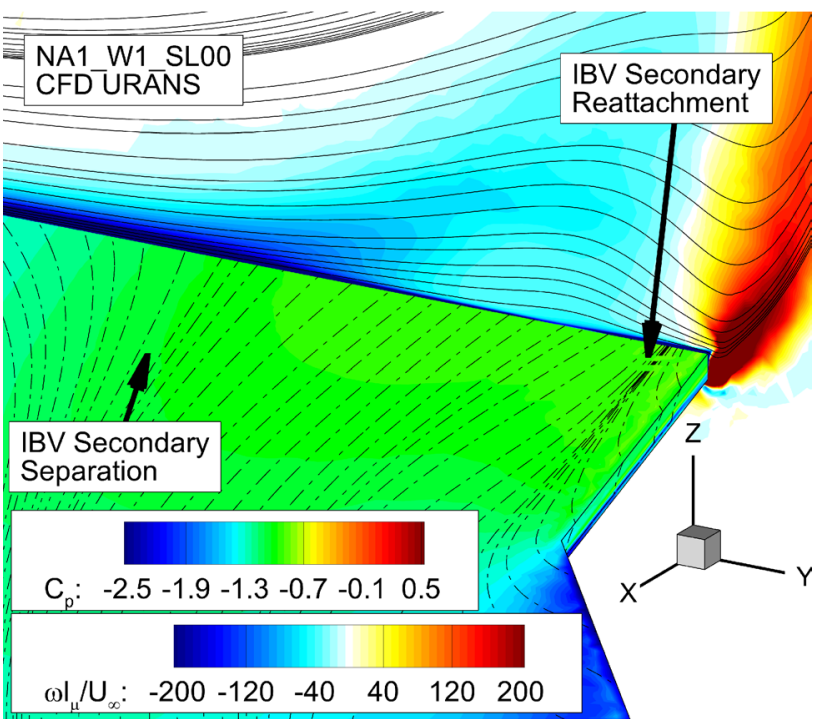

(a) URANS

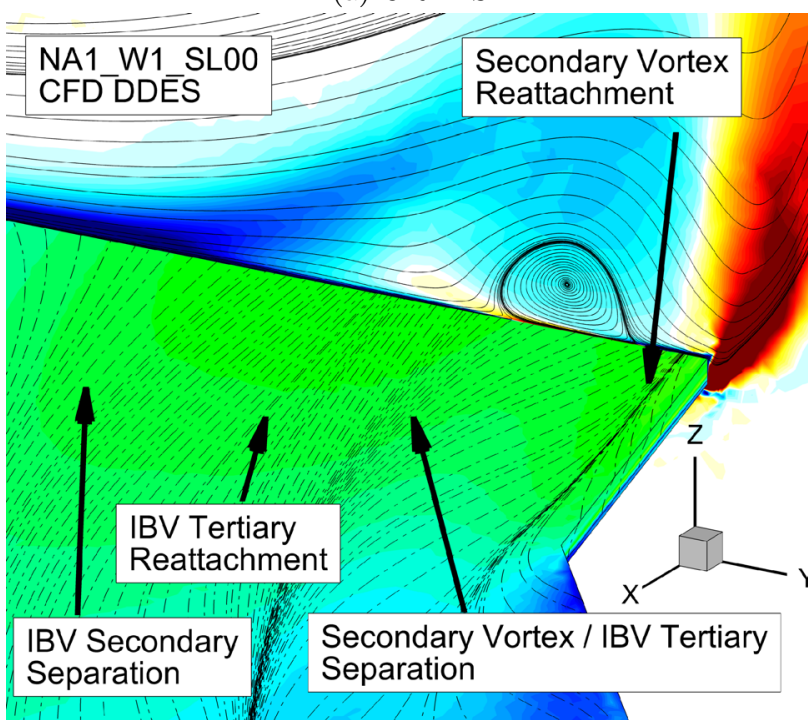

(b) DDES

Fig. 11 Non-dimensional axial vorticity $\omega l_{\mu} / U_{\infty}$ and flow streamlines (solid) at $x / c_{\mathrm{r}}=0.45$ as well as surface pressure distribution $C_{p}$ and skin friction lines (dash-dotted) at $\alpha=16^{\circ}$ and $\mathrm{Re}=3.0 \times 10^{6}$ for the NA1 W1 configuration

\subsection{Double-delta wing - NA1 W2}

\subsubsection{Forces and moments}

The lift and pitching moment coefficients for the NA1 W2 configuration are displayed in Fig. 12 in the same way as for the NA1 W1 configuration. The measured lift coefficient shows a non-linear increase in lift for angles of attack beyond $\alpha=8^{\circ}$. At $\alpha \approx 23^{\circ}$, the lift collapses abruptly by $\Delta C_{\mathrm{L}} \approx 0.1$. This indicates a breakdown of the vortex system [19]. The maximum lift of $C_{\mathrm{L}} \approx 1.41$ is reached at $\alpha \approx 33^{\circ}$. 


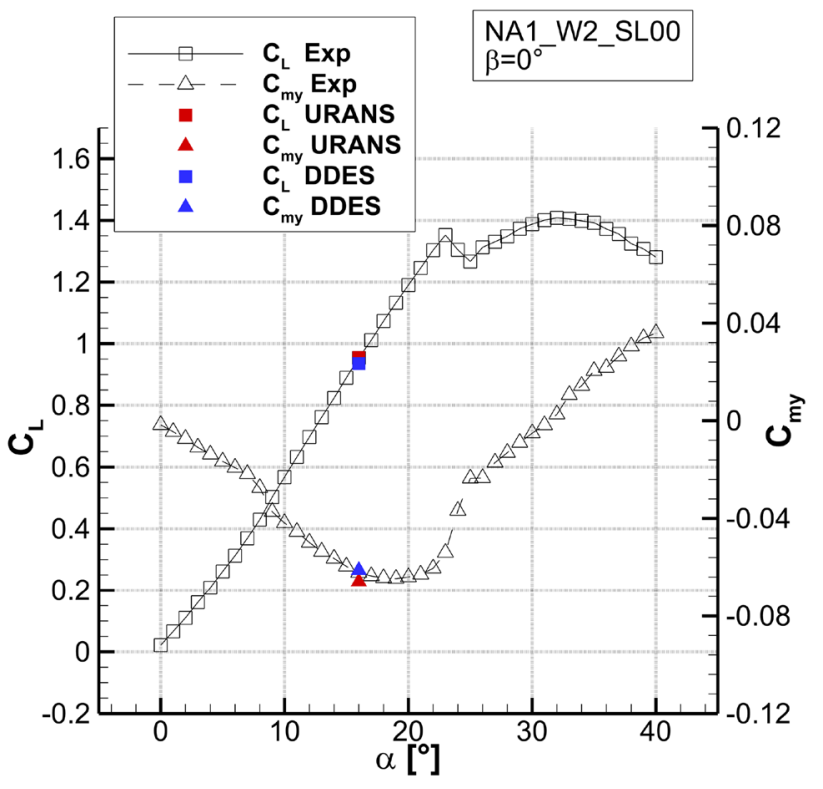

Fig. 12 Comparison of lift coefficient $C_{\mathrm{L}}$ and pitching moment coefficient $C_{m y}$ for NA1 W2 between experiments, URANS and DDES results at $\operatorname{Re}=3.0 \times 10^{6}$

The results of the URANS simulations show a good agreement with the experiments. For the DDES simulation for $\alpha=16^{\circ}$, a very high accuracy applies to both the lift and pitching moment coefficients with respect to the experimental data.

In Table 5, the coefficients for $\alpha=16^{\circ}$ are presented. The deviation of the URANS results from the experimental ones is very low and is even slightly lower than the deviation of the DDES results. Compared to the NA1 W1 results the URANS simulation is in better agreement with the experimental data, especially, regarding the pitching moment coefficient.

\subsubsection{Flow field}

For the comparison of the flow field of the NA1 W2 configuration, various crossflow sections are shown in Fig. 13 for PIV (left), URANS (center) and DDES (right). Due to the strake part of the wing planform, the first cross section was set at $x / c_{\mathrm{r}}=0.237$, cf. Fig. 4b. At $x / c_{\mathrm{r}}=0.237$, the IBV forms with a core velocity peak of $u / U_{\infty}=1.86$, indicating a jet-type vortex in the PIV results. The velocities in the vortex core in the URANS solution, $u / U_{\infty}=2.5$, and the DDES results, $u / U_{\infty}=2.3$, show a similar structure, but the simulation with URANS predicts the core flow velocitiy significantly too high. For $x / c_{\mathrm{r}}=0.475$, the IBV has increased in diameter and moved away from the wing surface. The core peak velocities for PIV, $u / U_{\infty}=2.06$, URANS, $u / U_{\infty}=2.62$, and DDES, $u / U_{\infty}=1.98$, show the same behavior as in the first cross section. In the next
Table 5 Absolute value and deviation of lift and pitching moment coefficients for $\alpha=16^{\circ}$ and $\mathrm{Re}=3.0 \times 10^{6}$ for NA1 W2 configuration

\begin{tabular}{llllll}
\hline & \multicolumn{1}{l}{$C_{\mathrm{L}}$} & & \multicolumn{2}{l}{$C_{m y}$} \\
\cline { 2 - 3 } \cline { 5 - 6 } & - & $\%$ & & - & $\%$ \\
\hline EXP & 0.954 & - & -0.0622 & - \\
URANS & 0.955 & $+0.126 \%$ & -0.0658 & $-5.79 \%$ \\
DDES & 0.948 & $-0.63 \%$ & -0.0612 & $+1.61 \%$ \\
\hline
\end{tabular}

cross section, at $x / c_{\mathrm{r}}=0.592$, two velocity maxima are visible. The left with an axial velocity peak of $u / U_{\infty}=2.22$ for PIV, $u / U_{\infty}=2.58$ for URANS and $u / U_{\infty}=1.78$ for DDES, exhibits an accelerating flow in the IBV core. The right velocity maximum represents the jet-type vortex core of the MBV. The URANS simulations predicts the core velocities too high and the DDES indicates a too dissipativ behavior with lower velocities compared to the experiment. At $x / c_{\mathrm{r}}=0.650$, the cores of the vortices approach each other, starting a strong vortex interaction. This initiates the rotation of the MBV above the IBV, visible in the next cross section, at $x / c_{\mathrm{r}}=0.767$. The velocity maxima fuse in the PIV and DDES results. In the URANS simulation two discrete maxima are still clearly visible. Additionally, the MBV in the URANS simulation has rotated farther inboard, compared to the other results. The occuring velocity maxima range between $u / U_{\infty}=2.62$ for URANS and $u / U_{\infty}=1.76$ for DDES. The axial peak velocity illustrated in the PIV results, $u / U_{\infty}=2.38$, is closer to the URANS simulations result. The same holds for the most downstream cross section, located at $x / c_{\mathrm{r}}=0.825$. The vortices have performed a full rotation, placing the MBV more inboard and the IBV in the outboard region. The rotation of the IBV underneath the MBV causes the separation of a secondary vortex. The flow fields shown by PIV and URANS are quite similar, disregarding the higher core velocities in the URANS results and the slightly divergent MBV core positions. The DDES simulation shows minor accelerated flow and has a large discrepancy to the other methods. In summary, the URANS is predicting jet-type vortices as more stable and with higher core velocities than the experiments. The DDES as well is in good agreement with the experiments but exhibits significantly lower core velocities.

In Fig. 14, the isosurfaces of the $Q^{*}$-criterion, $Q^{*}=50$, is shown colored by the total pressure ratio $p_{t} / p_{t, \infty}$ as well as the isosurface of the axial velocity $u / U_{\infty}=0$, in black. The positions of the flow field cross sections shown above, c.f. Fig. 13, are indicated by black lines on the model. The DDES results are shown on the left side and the URANS simulation on the right side. The development of the IBV is presented similarly by both methods. The LEV related shear layer is visible and is surrounding the downstream expanding IBV. After the change in wing sweep the URANS 

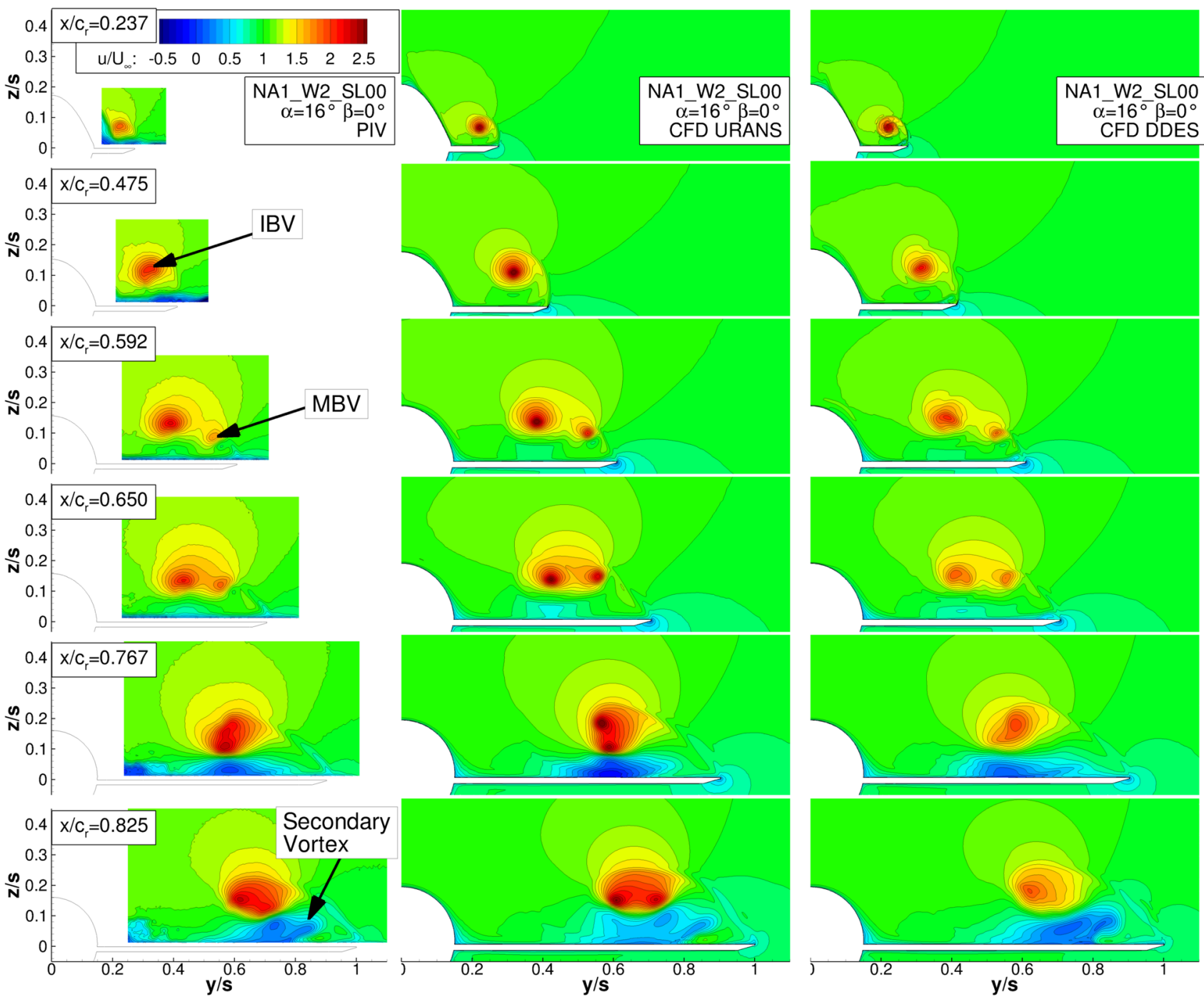

Fig. 13 PIV, URANS and DDES results of non-dimensional axial velocities $u / U_{\infty}$ at $\alpha=16^{\circ}$ and $\operatorname{Re}=3.0 \times 10^{6}$ for cross section planes ranging from $x / c_{\mathrm{r}}=0.237$ to $x / c_{\mathrm{r}}=0.825$ for NA1 W2 configuration

results indicate the rotation of the MBV around the IBV starts more upstream and the rotation is faster compared to the experimental data. The IBV as well as the MBV have a significantly smaller diameter in the URANS simulation, indicating a stronger, more stable vortex with a lower core pressure. At $x / c_{\mathrm{r}} \approx 0.80$, an area of $u / U_{\infty}=0$ appears above the wing surface due to the high deflection of the IBV close to the surface. Due to the mutual influence of the vortices, the vortex axes are significantly curved and therefore, induce reverse flow close to the wing surface. Downstream of $x / c_{\mathrm{r}}=0.825$, the vortex breaks down in the DDES. The $Q^{*}$ isosurface shows an expanded vortex field and rapidly increasing pressure. The URANS indicates breakdown more downstream and show reverse flow in the burst area. The vortex burst in the DDES solution is characterized by higher turbulence levels but no reverse flow effects occur.
In contrast to the NA1 W1 configuration, both IBV and MBV form as jet-type vortices and the vortex breakdown is more abrupt. Additionally, the breakdown position varies between the numerical methods.

In Fig. 15, the vortex core trajectories are shown for URANS (blue) and DDES (black). The positions of the IBV core are quite similar in the front region. As for the NA1 W1 configuration, the IBV core position in the URANS results is slightly closer to the leading edge.

Until the development of the MBV, the IBV trace is almost linear. Due to the vortex interaction the IBV moves in outboard direction and closer to the wing surface. The MBV rotates above the IBV directed inboard. Comparing the numerical methods, the URANS results show a stronger interaction between the vortices and a faster 


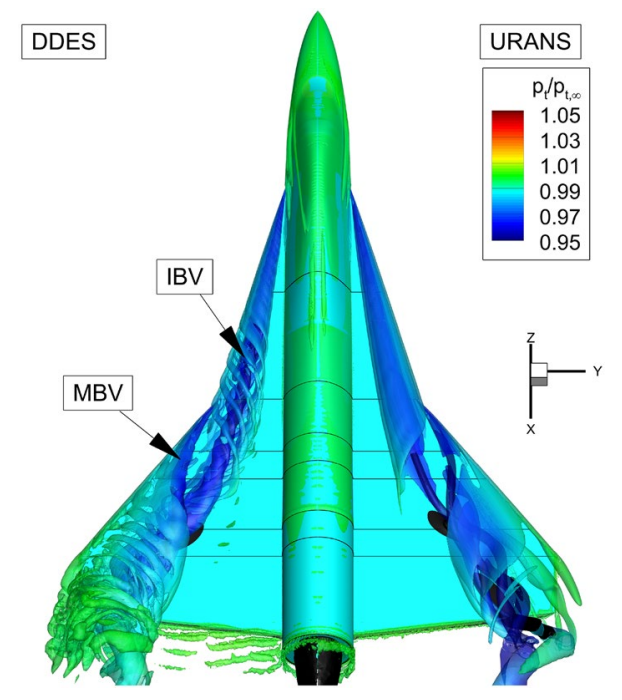

Fig. 14 Isosurfaces of the $Q^{*}$-criterion, $Q \times l_{\mu}^{2}=50$, colored with the total pressure ratio $p_{t} / p_{t, \infty}$, and $U / U_{\infty}=0$ (black) for DDES (left) and URANS (right) at $\alpha=16^{\circ}$ and $\mathrm{Re}=3.0 \times 10^{6}$ for NA1 W2 configuration

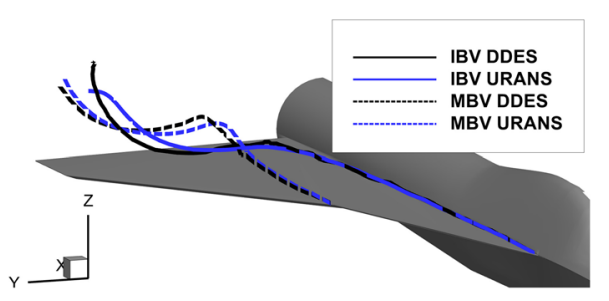

Fig. 15 Vortex core position for URANS (blue) and DDES (black) simulation determined by the $Q^{*}$-criterion at $\alpha=16^{\circ}$ and $\operatorname{Re}$ $=3.0 \times 10^{6}$ for $\mathrm{NA} 1 \mathrm{~W} 2$ configuration

rotation. Due to the weaker vortices the DDES results show less interaction between the vortices.

\subsubsection{Near-wall flow}

To describe the near-wall flow phenomena, the oil flow picture of the NA1 W2 configuration is presented in Fig. 16a. In addition, the surface pressure coefficient distributions for both numerical methods are also shown in Fig. 16. The streamlines in Fig. 16a indicate a quite similar picture as in Fig. 9a for the NA1 W1 configuration. In the front section, the IBV develops and is characterized by the strongly curved streamlines underneath the vortex. A secondary and tertiary structure is also present. The divergence streamline of the primary vortex is located close to the fuselage. A complex flow field with additional convergence and divergence lines due to the MBV is shown directly downstream of the change of the wing sweep at $x / c_{\mathrm{r}}=0.475$. The secondary and tertiary IBV separation lines merge with the MBV secondary

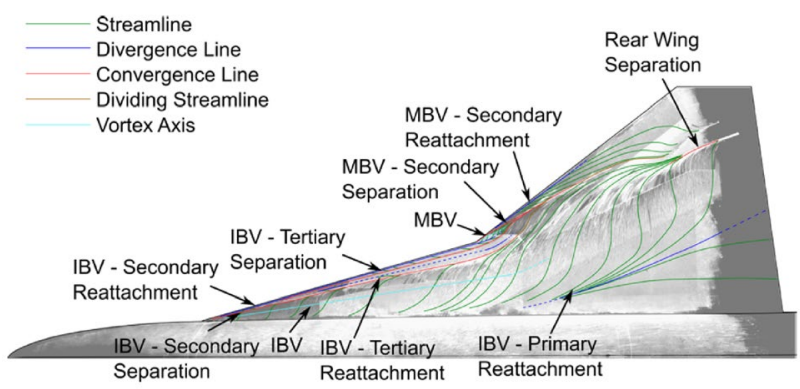

(a) Oil flow picture and inferred wall stream lines [19]

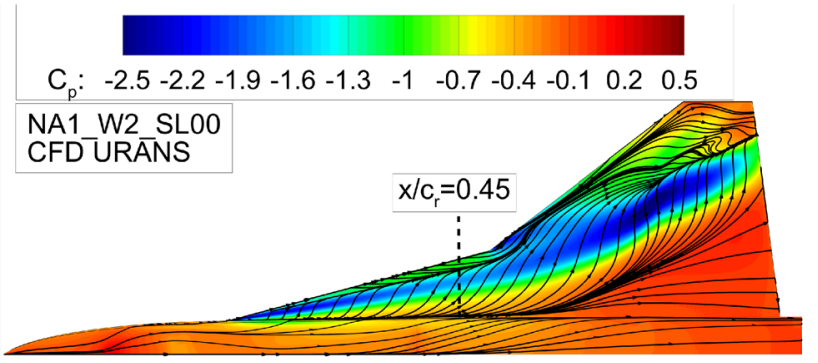

(b) Surface pressure distribution $C_{p}$ and skin friction lines of URANS solution

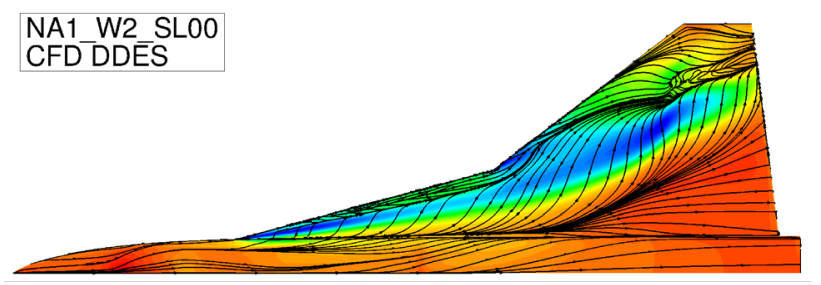

(c) Surface pressure distribution $C_{p}$ and skin friction lines of DDES solution

Fig. 16 Experimental and numerical results of the near-wall flow at $\alpha=16^{\circ}$ and $\operatorname{Re}=3.0 \times 10^{6}$ for NA1 W2 configuration

separation. This indicates a separation of the MBV from the wing surface. In the rear section, the attachment line of the stable vortex system is moving outwards and a further separation line is developing in the rear wing area. Due to low skin friction in the region downstream and outboard of the rear separation line, the wall near flow could not be resolved experimentally [19].

A similar behavior is shown by the numerical results of URANS, see Fig. 16b, and DDES, see Fig. 16c. Two major differences occur in these results. In the URANS solution there is only a secondary vortex structure detectable. The separation line of the IBV secondary structure does not converge with the separation line of the MBV as it is shown in the oil flow picture. Additionally, a reverse flow effect is present in the rear wing area. Due to the reverse flow the streamlines outboard of the separation line move back inboard.

Compared to the URANS solution a secondary as well as a tertiary IBV structure can be recognized in the DDES result. The separation lines of these structures converge with 
the MBV separation line. This is the only separation line in the region between $x / c_{\mathrm{r}}=0.55$ and $x / c_{\mathrm{r}}=0.8$. Upstream of $x / c_{\mathrm{r}}=0.825$, the skin friction lines divide in a separation and a reattachment line, indicating the development of a secondary vortex. This behavior is also indicated in Fig. 13.

The pressure distributions show generally the same structure, but the URANS solution provides with $C_{\mathrm{p}, \min }=-2.92$ a more pronounced pressure minimum than DDES, $C_{\mathrm{p}, \min }=-2.74$. Especially at $x / c_{\mathrm{r}}=0.767$, where the IBV is directly underneath the MBV, the pressure distribution varies strongly, between URANS, $C_{p}=-2.70$, and DDES, $C_{p}=-2.15$. These reduced suction levels also explain the lower lift coefficient in Table 5. In both cases, the pressure distribution in the front part shows a suction peak, that is caused by the developing IBV. More downstream the suction peak is decreasing due to movement of the IBV away from the wing surface. At $x / c_{\mathrm{r}}=0.475$, the development of the MBV increases the suction peak of the IBV and has its minimum at $x / c_{\mathrm{r}}=0.767$ due to the rotation of the MBV around the IBV. Due to this rotation a separation occurs and causes a second pressure minimum, $C_{p}=-0.91$, in the URANS results at $x / c_{\mathrm{r}}=0.825$ outboard of the peak of the main vortex system, $C_{p}=-2.35$. In the DDES solution, the breakdown of the vortex system is shown by a rapidly increasing surface pressure.

In Fig. 17, the pressure distributions in selected slices, $x / c_{\mathrm{r}}=0.237,0.475,0.592,0.825$, are shown. In the first slice, in black, the surface pressures of URANS and DDES simulations are quite similar, disregarding the lower pressure of the secondary vortex for the DDES. In the slice at $x / c_{\mathrm{r}}=0.592$, the discrepancy between the methods increases and the IBV suction is higher in the URANS results. The suction peak of the MBV is on the same level. In the last slice, the IBV suction peak of the URANS simulation is higher than in the DDES. This is also comparable

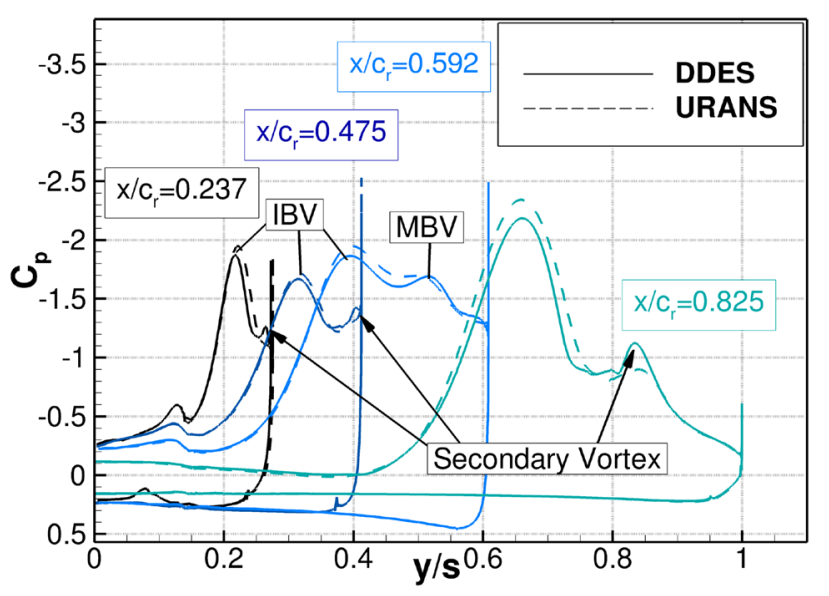

Fig. 17 Surface pressure distribution $C_{p}$ for selected sections at $\alpha=16^{\circ}$ and $\operatorname{Re}=3.0 \times 10^{6}$ for NA1 W2 configuration

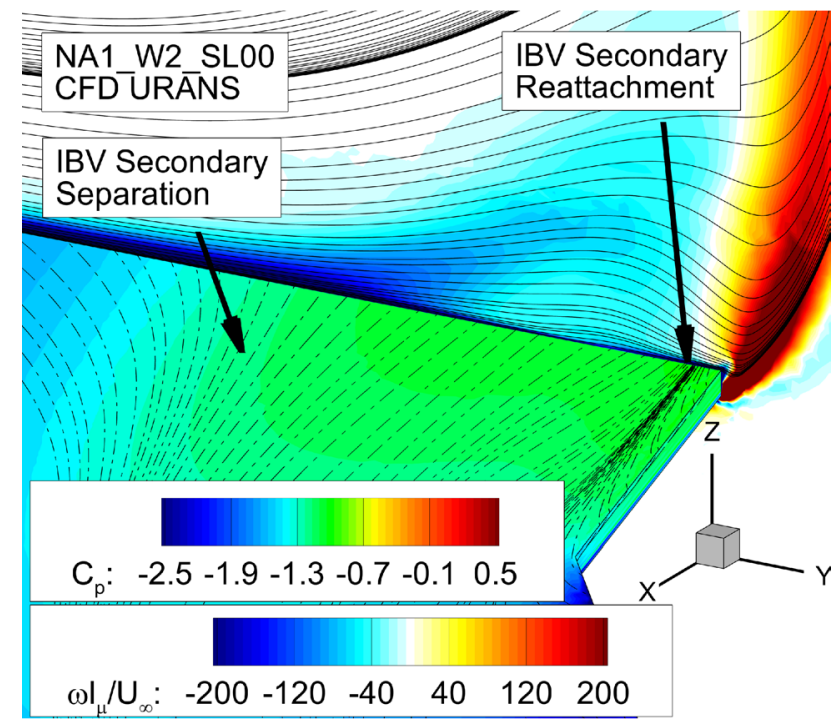

(a) URANS

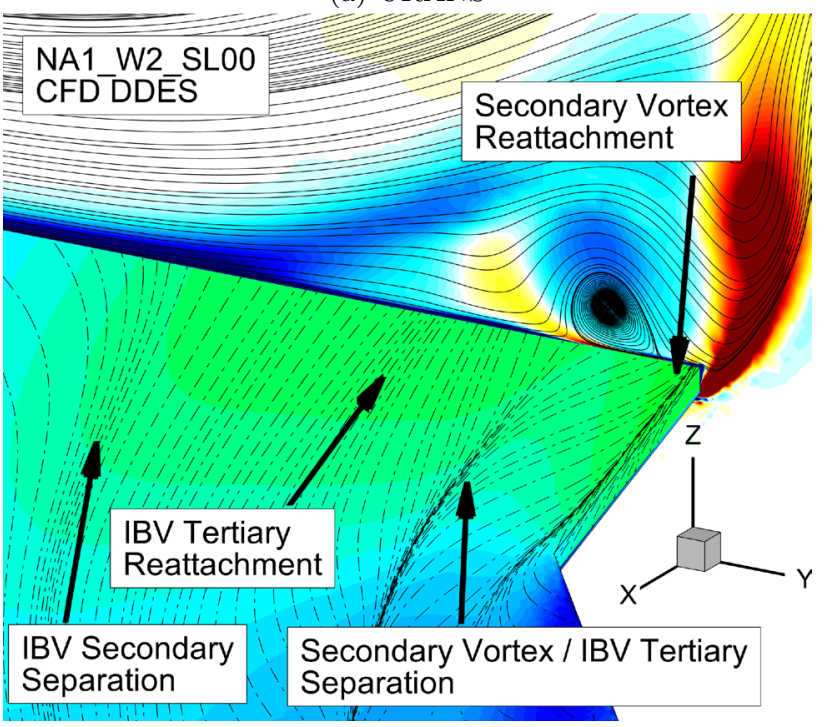

(b) DDES

Fig. 18 Non-dimensional axial vorticity $\omega l_{\mu} / U_{\infty}$ and flow streamlines (solid) at $x / c_{\mathrm{r}}=0.45$ as well as surface pressure distribution $C_{p}$ and skin friction lines (dotted) $\alpha=16^{\circ}$ and $\mathrm{Re}=3.0 \times 10^{6}$ for NA1 W2 configuration

to Fig. 13, showing similar structures in the front region but a decrease of axial velocity in the DDES results in the rear section. Especially downstream of $x / c_{\mathrm{r}}=0.825$, the surface pressure increases in the DDES results due to the more upstream vortex breakdown. The peak of the secondary vortex in the rear wing is considerably better resolved by the DDES simulation compared to the URANS results.

Figure 18 shows the development of the secondary vortex at $x / c_{\mathrm{r}}=0.45$. The crossflow is colored with the distribution of the non-dimensional axial vorticity $\omega l_{\mu} / U_{\infty}$ and the streamlines are displayed with solid lines. The wing 
surface illustrates the surface pressure coefficient and the skin friction lines presented by dash-dotted lines.

In Fig. 18a, the vorticity of the URANS result indicates a rotation in the opposite direction of the primary vortex, IBV. The streamlines separate from the surface but no vortical structure is visible. The skin friction lines indicate the same behavior. The IBV has a secondary separation and close to the leading-edge the flow reattaches on the wing surface.

The DDES results show a comparable behavior to the NA1 W1 configuration. The vorticity level of the secondary vortex is significantly higher, compared to the URANS simulation results. The secondary vortex causes a tertiary separation, rotating in the primary vortex direction and shown by positive axial vorticity. This behavior is also depicted in the skin friction lines. Starting from the left side, the primary vortex causes a secondary separation forming a secondary vortex close to the leading edge. The secondary reattachment line is moved closer to the leading edge, compared to the URANS results. The secondary vortex shares the separation line with the tertiary vortex. The tertiary reattachment line is displayed by slightly diverging skin friction lines left of the secondary vortex separation. The surface pressure indicates a significant pressure decrease due to the considerably stronger developed secondary vortex. This can also be seen in Fig. 17. The stronger secondary vortex also causes the slightly inboard shift of the DDES IBV core position, shown in Fig. 15.

In summary, the high resolution of the secondary vortex is important for a good agreement of the numerical and the experimental flow field. For the trajectory of the primary vortex and for the forces and moments, only a minor influence can be detected. For the latter, the vortex interaction and the breakdown behavior are more important.

\section{Conclusion}

A triple- and double-delta-wing-fuselage configuration was investigated for symmetric free stream at an angle of attack of $\alpha=16^{\circ}$ at $\operatorname{Re}=3.0 \times 10^{6}$ based on the reference length of $l_{\mathrm{Re}}=1 \mathrm{~m}$. Unsteady Reynolds-averaged Navier-Stokes and delayed detached eddy simulations were used as numerical methods. An analysis and comparison of longitudinal aerodynamic coefficients, flow field and near-wall flow have been presented for experimental and numerical data.

For the triple-delta wing, the DDES results show a very good agreement to the experimental data. The deviation of the pitching moment coefficient is significantly lower than the deviation of the URANS results. The flow field indicates a very good similarity with the PIV results.

The URANS forces and moments show a good accordance with the experimental data. The biggest issue is the velocity field of the vortex core flow with reverse flow even in the front wing area. The used setup has difficulties in the prediction of the flow structure related to wake-type vortex cores. The near-wall flow of the DDES is significantly closer to the experimental data, showing secondary and tertiary separation in the strake section of the wing. The results for the triple-delta-wing configuration indicates that the DDES attains results closer to the experiment compared to the URANS simulation.

For the double-delta-wing configuration, the URANS simulation show accurate predictions for the aerodynamic coefficients and the flow field for $\alpha=16^{\circ}$ but also for higher angles of attack. The flow field is close to that of the experiment and predicts only higher velocities in the vortex core. The DDES provides the aerodynamic coefficients at the same level of accuracy as the URANS simulations. But in the flow field the velocities in the vortex core are illustrated too low. Similar to the triple-delta-wing case the near-wall flow is predicted more precisely and shows tertiary structures. URANS and DDES are on the same accuracy level on that case based on comparison with the experimental data.

Comparing the two flow scenarios URANS-SA computations provide reliable results for stable jet-type LEV and LEV breakdown due to a strong adverse pressure rise.

DDES computations substantiate their superiority for wake-type LEVs and vortex breakdown cases associated with moderate adverse pressure gradients resulting in complex multiple vortex systems.

Further simulations on higher angles of attack and on angles of sideslip will be conducted to extend the comprehesion of the flow specific dependencies of the turbulence modelling.

Acknowledgements The project is funded by the Deutsche Forschungsgemeinschaft (DFG, German Research Foundation) - grant number BR1511/13-1. The fruitful cooperation with Airbus Defense and Space is gratefully acknowledged. Furthermore, the authors thank the German Aerospace Center (DLR) for providing the DLR TAU-Code used for the numerical investigations. Moreover, the authors gratefully acknowledge the Gauss Centre for Supercomputing e.V. (http://www.gausscentre.eu) for funding this project by providing computing time on the GCS Supercomputer SuperMUC at Leibniz Supercomputing Centre (http://www.lrz.de). The authors also highly appreciate the previous wind tunnel tests by Stefan Pfnür.

Funding Open Access funding enabled and organized by Projekt DEAL.

Open Access This article is licensed under a Creative Commons Attribution 4.0 International License, which permits use, sharing, adaptation, distribution and reproduction in any medium or format, as long as you give appropriate credit to the original author(s) and the source, provide a link to the Creative Commons licence, and indicate if changes were made. The images or other third party material in this article are included in the article's Creative Commons licence, unless indicated otherwise in a credit line to the material. If material is not included in the article's Creative Commons licence and your intended use is not permitted by statutory regulation or exceeds the permitted use, you will 
need to obtain permission directly from the copyright holder. To view a copy of this licence, visit http://creativecommons.org/licenses/by/4.0/.

\section{References}

1. Allmaras, S., Johnson, F., Spalart, P.: Modifications and clarifications for the implementation of the Spalart-Allmaras turbulence model. In: Seventh International Conference on Computational Fluid Dynamics (ICCFD7) (2012)

2. Breitsamter, C.: Unsteady flow phenomena associated with leading-edge vortices. Prog. Aerosp. Sci. 44(1), 48-65 (2008). https:// doi.org/10.1016/j.paerosci.2007.10.002

3. Brennenstuhl, U., Hummel, D.: Vortex formation over doubledelta wings. In: 13th Congress of the International Council of the Aeronautical Sciences, International Council of the Aeronautical Sciences Paper 6.6.3, Amsterdam, The Netherlands, pp. 1133$1146(1982)$

4. Buzica, A., Biswanger, M., Breitsamter, C.: Detached Eddy-simulation of delta-wing post-stall flow control. Transp. Res. Procedia 29, 46-57 (2018). https://doi.org/10.1016/j.trpro.2018.02.005

5. Buzica, A., Breitsamter, C.: Turbulent and transitional flow around the avt-183 diamond wing. Aerosp. Sci. Technol. 92, 520-535 (2019). https://doi.org/10.1016/j.ast.2019.06.025

6. Cummings, R.M., Morton, S.A., McDaniel, D.R.: Experiences in accurately predicting time-dependent flows. Prog. Aerosp. Sci. 44(4), 241-257 (2008). https://doi.org/10.1016/j.paerosci.2008. 01.001

7. Frink, N., Tomac, M., Rizzi, A.: Collaborative study of incipient separation on 53deg swept diamond wing. Aerosp. Sci. Technol. 57, 76-89 (2016)

8. Goertz, S.: Detached-Eddy simulations of a full-span delta wing at high incidence. In: 21st AIAA Applied Aerodynamics Conference (2003). https://doi.org/10.2514/6.2003-4216

9. Gursul, I.: Review of unsteady vortex flows over slender delta wings. J. Aircr. 42(2), 299-319 (2005). https://doi.org/10.2514/1. 5269

10. Hitzel, S.: Flightphysical aspects and methods of future military aircraft designs. CEAS Paper, vol. 119 (2015)

11. Hitzel, S.: Sub- and transonic vortex breakdown flight condition simulations of the F-16XL aircraft. J. Aircr. 31(4), 868-878 (2016). https://doi.org/10.2514/3.46573

12. Hummel, D., John, H., Staudacher, W.: Aerodynamic characteristics of wing-body-combinations at high angles of attack. In: 14th Congress of the International Council of the Aeronautical Sciences, International Council of the Aeronautical Sciences Paper 2.7.1, Amsterdam, Netherlands, pp. 747-762 (1984)

13. Kolác, V.: Vortex identification: new requirements and limitations. Int. J. Heat Fluid Flow 28(4), 638-652 (2007). https://doi.org/10. 1016/j.jijheatfluidflow.2007.03.004
14. Luckring, J.M., Park, M.A., Hitzel, S.M., Jirásek, A., Lofthouse, A.J., Morton, S.A., McDaniel, D.R., Rizzi, A., Tomac, M.: Synthesis of hybrid computational fluid dynamics results for $\mathrm{f}-16 \mathrm{xl}$ aircraft aerodynamics. J. Aircr. 54(6), 2100-2114 (2017). https:// doi.org/10.2514/1.C034053

15. Morton, S., Forsythe, J., Mitchel, A., Hajek, D.: DES and RANS simulations of delta wing vortical flows. AIAA 2002-0587 (2002). https://doi.org/10.2514/6.2002-587

16. Nitsche, W., Brunn, A.: Strömungsmesstechnik. Springer, Berlin (2006). https://doi.org/10.1007/3-540-32487-9

17. Pfnür, S., Breitsamter, C.: Leading-edge vortex interactions at a generic multiple swept-wing aircraft configuration. J. Aircr. 56(6), 2093-2107 (2019). https://doi.org/10.2514/1.C035491

18. Pfnür, S., Breitsamter, C.: Stereo-PIV measurements of vortexinteraction effects on generic delta wing planforms. In: 13th International Symposium on Particle Image Velocimetry-ISPIV 2019 (2019)

19. Pfnür, S., Hövelmann, A., Sedlacek, D., Breitsamter, C.: Vortex flow interaction phenomena on multi swept delta wings at subsonic speeds. STO-MP-AVT-307, 20 (2019)

20. Raffel, M., Willert, C.E., Wereley, S.T., Kompenhans, J.: Particle Image Velocimetry-A Practical Guide. Springer, Berlin (2007)

21. Sciacchitano, A., Wieneke, B.: PIV uncertainty propagation. Meas. Sci. Technol. (2016). https://doi.org/10.1088/0957-0233/ 27/8/084006

22. Shur, M.L., Spalart, P.R.: On the sensitization of turbulence models to rotation and curvature. AIAA J. 38(5), 784-792 (1997). https://doi.org/10.2514/2.1058

23. Shur, M.L., Strelets, M.K., Travin, A.K., Spalart, P.R.: Turbulence modeling in rotating and curved channels: assessing the SpalartShur correction. AIAA J. 38(5), 784-792 (2000). https://doi.org/ $10.2514 / 2.1058$

24. Spalart, P., Allmaras, S.: A one-equation turbulence model for aerodynamic flows. AIAA (1992)

25. Spalart, P.R.: Young-persons guide to detached-eddy simulation grids, pp. 2001-211032. Technical report, NASA CR (2001)

26. Spalart, P.R.: Detached-Eddy simulation. Annu. Rev. Fluid Mech. 41(1), 181-202 (2009). https://doi.org/10.1146/annurev.fluid. 010908.165130

27. Spalart, P.R., Deck, S., Shur, M., Squires, K., Strelets, M., Travin, A.: A new version of detached-Eddy simulation, resistant to ambiguous grid densities. Theor. Comput. Fluid Dyn. 20, 181-195 (2006). https://doi.org/10.1007/s00162-006-0015-0

Publisher's Note Springer Nature remains neutral with regard to jurisdictional claims in published maps and institutional affiliations. 